\title{
Bounding techniques for transient analysis of G-Networks with catastrophes
}

\author{
Hind Castel-Taleb \\ Telecom SUDPARIS \\ 9 ,rue Charles Fourier \\ 91011 Evry Cedex, France \\ hind.castel@it- \\ sudparis.eu
}

\author{
Idriss Ismael-Aouled \\ LACL, Univ. Paris-Est Créteil \\ 61, av. du Général de Gaulle \\ 94010 Créteil Cedex, France \\ idriss.ismael@it- \\ sudparis.eu
}

\author{
Nihal Pekergin \\ LACL, Univ. Paris-Est Créteil \\ 61, av. du Général de Gaulle \\ 94010 Créteil Cedex, France \\ nihal.pekergin@u-pec.fr
}

\begin{abstract}
We apply stochastic comparisons in order to bound the transient behavior of G-networks with catastrophes. These networks belong to Gelenbe's networks, with both positive and negative customers (or signals). We consider catastrophes where the signal deletes all customers in a queue. G-networks have a known product form steady-state distribution, but it is still impossible to obtain the transient distributions by a closed form. In the present paper, we propose to define smaller queueing systems providing bounds for subnetworks of the G-network with catastrophes. We apply stochastic comparisons by mapping functions to build bounding models. We derive transient performance measure bounds for applications as malware software infections. For instance, we obtain bounds for the first time of infection, or the number of times a station has been infected in a time interval. We study the tradeoff between the size of the subnetwork and the quality of the bounds with respect to parameters.
\end{abstract}

\section{Keywords}

G-Networks, transient behavior, analytical methods, stochastic comparisons

\section{INTRODUCTION}

Since the seminal papers by Gelenbe in the early nineties $[10$, $11,12]$, Generalized networks of queues have received considerable attention. Currently, there are several hundred references devoted to the subject, and a book [24] provides insight into some of the research issues, developments and applications in the area of networks of queues with customers and signals. Queueing models typically do not have provisions for some customers being used to eliminate other customers, or to redirect other customers among the queues. In other words, customers in traditional queueing networks cannot exert direct control on other customers. G-network models

* partially supported by french research project ANRSETI06-02

Permission to make digital or hard copies of all or part of this work for personal or classroom use is granted without fee provided that copies are not made or distributed for profit or commercial advantage and that copies bear this notice and the full citation on the first page. To copy otherwise, to republish, to post on servers or to redistribute to lists, requires prior specific permission and/or a fee.

VALUETOOLS 2011, May 16-20, Paris, France

Copyright (C) 2011 ICST 978-1-936968-09-1

DOI 10.4108/icst.valuetools.2011.245719 overcome some of the limitations of conventional queueing network models and still preserve the computationally attractive product form property of some Markovian queueing networks. They contain unusual customers such as negative customers which eliminate normal customers, catastrophes which flush all the customers out of a queue [12] and triggers which move other customers from one queue to another [11] or resets [15]. The first type of signal introduced by Gelenbe was described as a negative customer [10]. A negative customer deletes a positive customer in a queue at its arrival if it is possible. Positive customers are usual customers in classical queueing networks. A negative customer is never queued. Under typical assumptions (Poisson arrival for both types of customers, exponential service time for positive customers, Markovian routing, independence, open topology) Gelenbe proved that such a network has a product form solution for its steady-state behavior. Network of positive and negative customers were introduced to model neural networks where neurons exchange inhibitory and exciting signals [13]. Gnetworks are also used to model complex operations such as work deletion [1], or software virus infections [14].

Transient analysis of G-networks is very difficult as it is quite impossible to derive a closed form expression of the transient distribution. Stochastic comparisons provide interesting solutions for this problem since they lead to build bounds both on the stationary and the transient probability distributions. Massey has introduced in [20] some stochastic bounds for the transient tail distribution functions of the size of any queue in Jackson networks. The bounding model consists in independent $\mathrm{M} / \mathrm{M} / 1$ queues for which the transient probability distribution can be computed as in [17]. In [5], this approach is generalized to build bounding subnetworks for Jackson networks. In [23, 4], increasing set formalism has been applied to bound tail distribution functions of G-networks and G-networks with catastrophes by means of independent $\mathrm{M} / \mathrm{M} / 1$ queues. In this paper, we apply bounding techniques with a different approach in order to define more precise bounding systems. The bounding systems are indeed queueing systems but their state space sizes are smaller than the original one. In [4], the links are cut between all the queues, thus each queue of the original system is bounded by an $M / M / 1$ queue. Since we do not consider customer transfers between the queues, these bounding systems may be too far from the exact system. In the present paper, we follow the approach proposed in [5] for Jackson networks and build bounds in the presence 
of negative customers. We bound the G-subnetwork under study by a subnetwork of the same size but with different rates that are obtained using stochastic comparisons. The relevance of the approach is to keep the customer transfers between the queues inside the subnetwork.

We focus on a subnetwork of the G-networks with catastrophes, and we define bounding systems with the same size from which we compute performance measure bounds. Bounding systems are defined by cutting the links between the subnetwork and the rest of the network. The impact of the queues which are not in the subnetwork are taken into account by means of additional external flows of positive and negative customers to the subnetwork. These additional flows are differently defined for the upper bound and for the lower bound. Intuitively speaking, in the upper bound we have more positive customers and less flushes of queues, while in the lower bound we have more flushes of queues and less positive customers. By enlarging the size of the bounding subnetwork, we can have a a tradeoff between the size of the subnetwork and the quality of the bounds. However, stochastic comparisons used in this paper are not easy to establish because of the complex dynamics of G-networks. In fact, G-networks are not monotone due to synchronized departure of customers, and if we keep this event in the bounding systems, then they could be also not monotone. So the comparison of the processes will be checked for each comparable states $x$ and $y$ such $x \preceq y[22,21]$.

We apply stochastic comparisons by mapping functions [9] on a smaller state space in order to define the bounding systems. These techniques are similar to the bounding aggregations [3], but in our case the aggregated state space is not a subset of the whole state space, but another state space that is smaller than the original one. Moreover, the algorithm in [3] could not applied as G-networks are not monotone. In the case of totally ordered state spaces, the lumpability and stochastic comparisons have been combined to derive bounding Markov chains [26]. In our study, we define the partial order component by component on the state space in order to compare queues by queues the processes. Bounding aggregations are defined by mapping functions in another state space, which is smaller than the original one. This approach could be interesting if we want to study only a part of the network. The best known stochastic ordering is the strong stochastic ordering (denoted by $\preceq_{s t}$ ) corresponding to sample-path comparisons $[22,25]$. We apply the coupling method to establish the stochastic comparison of the processes, This corresponds indeed to compare the evolution of the processes due to the events [19]. The coupling of the processes can be also done by means of compensation of jumps for the processes such that realizations stay in a set $K$ [16]. In [9], the coupling technique for stochastic comparison of functions of Continuous Time Markov Chains (CTMCs) is presented and conditions are generated such that the coupled process stays in the given set $K$. Application of stochastic comparisons to G-networks requires some subtleties. In fact, we have to consider positive and negative customers, with several kinds of events. We apply the general coupling technique for the definition of bounding systems.

The paper is organized as following. In section 2 , we introduce more formally the model of G-networks with catas- trophes. In section 3, we give main definitions of stochastic comparisons of Markov processes using the coupling method. In section 4 , we apply the coupling by mapping functions in order to define bounding systems for a subnetwork of the G-Network with catastrophes. We give the main transient measures that could be derived from bounding systems. In section 5, we consider an application of software virus infection and we give performance measure bounds. Finally, achieved results are discussed in the conclusion and comments about further research items are given.

\section{G-NETWORKS WITH CATASTROPHES}

We assume that the arrivals of new positive customers follow independent Poisson processes with rate $\lambda_{i}^{+}$at queue $i$. Similarly, the arrivals of negative customers (or signals) follow independent Poisson processes with rate $\lambda_{i}^{-}$at queue $i$. Positive customers wait in the queue and eventually receive service if they are not deleted by a signal. The service are exponential with rate $\mu_{i}$ at queue $i$. Signals are not queued. When they enter a queue, they flush all the customers out of the queue. At the completion of its service at queue $i$, a positive customer moves to queue $j$ either as a positive customer or as signal depending of a Markovian routing. The routing matrices are $P^{+}$and $P^{-}$to describe the movement of positive customer from queue $i$ to queue $j$ and the probability that a customer leaving queue $i$ arrives at queue $j$ as a signal. Finally $d_{i}$ is the probability that a positive customer leaves queue $i$ at the completion of its service. As usual with G-networks, we assume that there is no self-loops in the routing matrices: i.e. for all $i, P^{+}(i, i)=0$ and $P^{-}(i, i)=0$. Finally the total probability law gives that for all queue $i$ : $\sum_{j=1}^{n} P^{+}(i, j)+\sum_{j=1}^{n} P^{-}(i, j)+d_{i}=1$. We denote by $\{X(t), t \geq 0\}$ the CTMC with infinitesimal generator $Q$, representing the evolution of the G-Network with catastrophes. From state $x \in \mathbb{N}^{n}$ the transitions are:

$$
\begin{aligned}
& \text { - } x \rightarrow x+e_{i} \text { with rate } \lambda_{i}^{+}, 1 \leq i \leq n . \\
& \text { - } x \rightarrow x-x_{i} e_{i} \text { with rate } \lambda_{i}^{-}, x_{i}>0,1 \leq i \leq n . \\
& \text { - } x \rightarrow x-e_{i} \text { with rate } \mu_{i} d_{i}, \text { if } x_{i}>0,1 \leq i \leq n . \\
& \text { - } x \rightarrow x-e_{j}+e_{i} \text { with rate } \mu_{j} P^{+}(j, i), \text { if } x_{j}>0,1 \leq \\
& \quad i, j \leq n \text {, where } i \neq j . \\
& \text { - } x \rightarrow x-e_{j}-x_{i} e_{i} \text { with rate } \mu_{j} P^{-}(j, i), \text { if } x_{j}>0, x_{i}> \\
& \quad 0,1 \leq i, j \leq n, \text { where } i \neq j .
\end{aligned}
$$

Under these assumptions, it is proved in [12] that the Gnetworks have a product form steady-state distribution. Since the model in [12] is based on the destruction of batches of customer by signals, it is more general and the queue flushing considered here is associated to an infinite batch. It is also (and independently) proved that G-networks with catastrophes have product form solution in [6]. The transient behavior of this system is very difficult to study. We propose to use stochastic comparisons by defining bounding systems such that the transient probability distribution is easier to compute. In the simple case where the subnetwork of the bounding system is represented by only one queue, then the transient probability distribution can be computed as it has been presented in the literature (see for instance 
$[17,7]$ and references therein). In the general case of multidimensional CMTCs, mathematical analysis and simulations could be applied for the computation of the transient probability distribution [2]. Furthermore, for large state space size, transient solutions are very difficult to obtain. Numerical methods allow to derive only approximations of the transient probability distributions. The relevance of this paper is to define bounding systems with a smaller size in order to derive easily transient probability distributions. These probability distributions represent bounds for the exact transient probability distribution on the considered subnetwork. All the computations are obtained from simulations. Next we present stochastic comparisons by mapping functions, using the coupling method.

\section{STOCHASTIC COMPARISONS}

We present some theorems and definitions about stochastic orderings used in this paper. Two formalisms can be used for the definitions: increasing functions $[22,25]$ or increasing sets [21]. Stochastic orderings are defined only on discrete and countable state space $A$, where a binary relation $\preceq$ represents at least a preorder (a reflexive and transitive binary relation [22]). Let us remark that a total or a partial order are also preorder relations. For instance, on $A=\mathbb{N}, \leq$ is a total order, and on $A=\mathbb{N}^{n}$, the component-wise order is a partial order, which are both also preorders. In the sequel of the paper, we suppose that random variables and stochastic processes take values on a state space $A$ endowed with a preorder $\preceq$. A stochastic ordering corresponds to an order relation between random variables or probability measures. The $\preceq_{s t}$-ordering is the best known stochastic ordering, it is equivalent to the sample path ordering (see Strassen's theorem [22]). The $\preceq_{s t}$-ordering can be defined using real increasing functions [22]:

Definition 1. $X \preceq_{s t} Y \Leftrightarrow E[(f(X))] \leq E[(f(Y))] \forall f:$ $A \rightarrow \mathbb{R}^{+}, \preceq$-increasing whenever the expectations exist.

These concepts are extended to the comparison of stochastic processes. Let $\{X(t), t \geq 0\}$ and $\{Y(t), t \geq 0\}$ be Continuous Time Markov Chains (CTMC)s defined on $A$. The stochastic comparison of CTMCs is equivalent to the comparison $\forall t \geq 0$ of the processes [22].

\section{DeFinition 2. We say that:}

$$
\{X(t), t \geq 0\} \preceq_{s t}\{Y(t), t \geq 0\} \text { if } X(t) \preceq_{s t} Y(t), \forall t \geq 0
$$

When the processes are defined on different state spaces, we can compare them on a common state space using mapping functions. This concept could be very interesting to reduce the state space by defining bounding aggregations [26, 3]. Let $X(t)$ (resp. $Y(t))$ defined on $A$ (resp. $B$ ), $g$ be a manyto-one mapping from $A$ to $B$. We suppose that state space $B$ is endowed with the preorder $\preceq$. We compare the mapping of the process $X(t)$, denoted $g(X(t))$ with the process $Y(t)$ on the common state space $B$. The stochastic comparisons of processes by mapping functions is defined as follows [9]:

Definition 3. We say that:

$$
\{g(X(t)), t \geq 0\} \preceq_{s t}\{Y(t), t \geq 0\} \text { if } g(X(t)) \preceq_{s t} Y(t), \forall t \geq 0 \text {. }
$$

The monotonicity is an interesting property because it simplifies the stochastic comparisons of CTMCs. It is defined as an increasing or decreasing in time [22]:

DEFINITION 4. $\{X(t), t \geq 0\}$ is said to be $\preceq_{s t}-$ monotone if $X(t) \preceq_{s t}\left(\succeq_{s t}\right) X(t+\tau), \forall t \geq 0, \forall \tau \geq 0$.

In this paper, we focus on the coupling method, used for the stochastic comparison in the sense of the $\preceq_{s t}$-ordering (called the $\preceq_{s t}$-comparison).

\subsection{The coupling method}

The coupling method is a well known method for comparisons of probability measures and Markov processes (see Lindvall [19]). First, we introduce the coupling of random variables. The coupling for the $\preceq_{s t}$-comparison of random variables is equivalent to the definition of a coupled version with a point-wise comparison:

TheOREM 1. For random variables $X$ and $Y$ with distribution functions $F_{X}$ and $F_{Y}$, the following statements are equivalent:

\section{1. $X \preceq s t Y$}

2. there is a coupling $(\widehat{X}, \widehat{Y})$ of $X$ and $Y$, and the probability space $(\Omega, \mathcal{A}, \mathcal{P})$ where $\widehat{X}$ and $\widehat{Y}$ are defined on it with the distribution functions $F_{X}$ and $F_{Y}$, such that:

$$
\widehat{X}[\omega] \preceq \widehat{Y}[\omega], \forall \omega \in \Omega
$$

In [16], the coupling is given between probability measures. If we denote by $P$ (resp. $P^{\prime}$ ) the probability measure of $X$ (resp. $Y$ ), then:

$$
P \preceq_{s t} P^{\prime}
$$

if and only if there exists a probability measure on the state space $A \times A$ with support in:

$$
K=\{(x, y) \in A \times A \mid x \preceq y\}
$$

with first marginal $P$, and second marginal $P^{\prime}$. It is a special case of Strassen's theorem [22]. The coupling method can be also applied for the $\preceq_{s t}$-stochastic comparison of Markov processes. It is equivalent to the definition of a coupled version of the processes in order to compare their sample paths. For the coupling of $\{X(t), t \geq 0\}$ and $\{Y(t), t \geq 0\}$, we define two other Markov processes on $A:\{\widehat{X}(t), t \geq 0\}$ and $\{\widehat{Y}(t), t \geq 0\}$ such that $\{\widehat{X}(t), t \geq 0\}$ has the same infinitesimal generator as $\{X(t), t \geq 0\}$, and $\{\widehat{Y}(t), t \geq 0\}$ the same infinitesimal generator as $\{Y(t), t \geq 0\}$. The coupling for the $\preceq_{s t}$-comparison of Markov processes is given in the following theorem (see Lindvall [19, 22]):

THEOREM 2. The following propositions are equivalent:

1. $\{X(t), t \geq 0\} \preceq s t\{Y(t), t \geq 0\}$ 
2. there exists the coupling $\{(\widehat{X}(t), \widehat{Y}(t)), t \geq 0\}$ such that $\forall t>0, \omega \in \Omega$ :

$$
\widehat{X}(0)[\omega] \preceq \widehat{Y}(0)[\omega] \Rightarrow \widehat{X}(t)[\omega] \preceq \widehat{Y}(t)[\omega]
$$

3. there exists the Markov process $\{Z(t)=(\widehat{X}(t), \widehat{Y}(t)), t \geq$ 0) taking values in

$$
K=\{(x, y) \in A \times A, x \preceq y\}
$$

Note that the $\preceq_{s t}$-comparison of Markov processes can be also established by means of inequalities between rows of the underlying generators $[16,22]$. The coupling is a method for the $\preceq_{s t}$-comparison of Markov processes based on the comparison of the evolution of sample paths due to events, which induces the comparison of transition rates of the processes. In the case where the processes are not defined on the same state space, we can apply the coupling by mapping functions [9]. Let $X(t)$ (resp. $Y(t)$ ) defined on $A$ (resp. $B$ ), with infinitesimal generator $Q^{X}$ (resp. $Q^{Y}$ ), and $g$ be a many-toone mapping from $A$ to $B$. Next, we give the theorem which states the coupling with the set $K$, and the inequalities on transition rates for the comparison of Markov processes by mapping functions $[9,21]$ :

THEOREM 3. The following propositions are equivalent:

1. $\{g(X(t)), t \geq 0\} \preceq_{s t}\{Y(t), t \geq 0\}$

2. $\forall \Gamma \in \Phi_{s t}(B), \forall g(x) \preceq y \mid g(x), y \in \Gamma$ or $g(x), y \notin \Gamma$, we have:

$$
\sum_{g(z) \in B} Q^{X}(x, z) \leq \sum_{z \in B} Q^{Y}(y, z)
$$

3. there exists the Markov process $\{Z(t)=(\widehat{X}(t), \widehat{Y}(t)), t \geq$ $0\}$ taking values in:

$$
K=\{(x, y) \in A \times B, g(x) \preceq y\}
$$

where $\Gamma$ is an increasing set of states in $\Phi_{s t}(B)$ which is the family of all increasing sets of $B[21,22]$. Note that this theorem can be also applied to check if

$$
\{Y(t), t \geq 0\} \preceq_{s t}\{g(X(t), t \geq 0\}
$$

by reversing the inequalities. In [9], the authors introduce the infinitesimal generator $Q$ of $Z(t)$, and they define the coupling process in order to obtain explicit conditions for stochastic comparisons. The Markov process $Z(t)$ stays always in $K$ after reaching $K$ if and only if the rates between $K$ and its complementary set, $K^{c}$ are null:

$$
Q\left[(x, y),\left(x^{\prime}, y^{\prime}\right)\right]=0, \forall(x, y) \in K, \forall\left(x^{\prime}, y^{\prime}\right) \in K^{c}
$$

The idea of the coupling is the following: if we are going out of $K$ by a jump of the first coordinate, we must balance it by a jump of the second coordinate in such a way that the final point is in $K$. In [9] necessary and sufficient conditions on the transition rates are derived for the stochastic comparison of Markov processes by mapping functions, using the coupling process $Z(t)$ associated to $K$. The coupling can be also used to establish the strong monotonicity. For $\{X(t), t \geq 0\}$, we apply the coupling of the process with itself ( see Lindvall [19]). So we define two processes: $\{\widehat{X}(t), t \geq 0\}$ and $\left\{\widehat{X^{\prime}}(t), t \geq 0\right\}$ governed by the same infinitesimal generator matrix as $\{X(t), t \geq 0\}$, representing different realizations of $\{X(t), t \geq 0\}$ with different initial conditions. The theorem of the monotonicity using the coupling is as follows (see Lindvall $[19,18])$ :

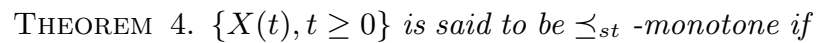
and only if there exists the coupling $\left\{\left(\widehat{X}(t), \widehat{X^{\prime}}(t)\right), t \geq 0\right\}$ such that:

$$
\widehat{X}(0)[\omega] \preceq \widehat{X^{\prime}}(0)[\omega] \Rightarrow \widehat{X}(t)[\omega] \preceq \widehat{X^{\prime}}(t)[\omega], \forall t>0
$$

Next, we give a simple example of the coupling by mapping functions in order to define an upper bounding process.

\subsection{Example}

Let take the following example of a queueing network modelled by 3 queues. For each queue $1 \leq i \leq 3$, the arrivals are Poisson with rates $\lambda_{i}$, the service times exponential with parameter $\mu_{i}$. The non null routing probabilities are : $P(1,3)=0.5, P(2,3)=0.5$, and the probabilities to go out from queues $d_{1}=0.5$, and $d_{2}=0.5$. This system can be represented by a CTMC $\{X(t), t \geq 0\}$, with infinitesimal generator $Q$. We propose to define the CTMC $\left\{X^{u}(t), t \geq 0\right\}$, with infinitesimal generator $Q^{u}$, such that $: g(X(t)) \leq_{s t} X^{u}(t)$ where $g$ is many-to-one mapping function $: g: \mathbb{N}^{3} \rightarrow \mathbb{N}$, such that $g\left(x_{1}, x_{2}, x_{3}\right)=x_{1}+x_{2}+x_{3}$, representing the total number of customers in the system. We will define $Q^{u}$ such that $\left(g(X(t)), X^{u}(t)\right)$ stays in the set $K=\left\{(x, y) \in \mathbb{N}^{3} \times \mathbb{N} \mid g(x) \leq y\right\}$. Obviously, we have two cases where $g(x)$ can be modified : it increases if we have an arrival in any queue $i$, and decreases if we have a service in any queue $i$. We will study now the jumps of the processes, and we define the inequalities between the transition rates in order to have the coupled process staying in set $K$.

1. If we have an arrival in any queue, then $x$ evolves to $x^{\prime}$ such that $g\left(x^{\prime}\right)=g(x)+1$. As we could have $g(x)+1>$ $y$ (if $x_{1}+x_{2}+x_{3}=y$ ), then we must have also an arrival in the second component $y$ in order to compensate it by a jump from $y$ to $y+1$, such that $g(x)+1 \leq y+1$. So the first component must increase less then the second one, and we derive the following inequality :

$$
\sum_{g\left(x^{\prime}\right)=g(x)+1} Q\left(x, x^{\prime}\right) \leq Q^{u}\left(y, y^{\prime}\right) .
$$

Since $\sum_{g\left(x^{\prime}\right)=g(x)+1} Q\left(x, x^{\prime}\right)=\lambda_{1}+\lambda_{2}+\lambda_{3}$, then we take $Q^{u}\left(y, y^{\prime}\right)=\lambda_{1}+\lambda_{2}+\lambda_{3}$.

2. if we have a service in $\left\{X^{u}(t), t \geq 0\right\}$, then $y$ evolves to $y^{\prime}$ such that $y^{\prime}=y-1$. So we must have a transit from $x$ to $x^{\prime}$ where $g\left(x^{\prime}\right)=g(x)-1$. We see that the first component must decrease more than the second one in order to obtain an upper bounding system.

$$
\sum_{g\left(x^{\prime}\right)=g(x)-1} Q\left(x, x^{\prime}\right) \geq Q^{u}\left(y, y^{\prime}\right) .
$$


Since $\sum_{g\left(x^{\prime}\right)=g(x)-1} Q\left(x, x^{\prime}\right)=\sum_{i=1}^{3} \mu_{i} d_{i} 1_{x_{i}>0}$, then we take $Q^{u}\left(y, y^{\prime}\right)=\min _{1 \leq i \leq 3} \mu_{i} d_{i}$.

So $\left\{X^{u}(t), t \geq 0\right\}$ is a birth and death process with birth rates $\lambda_{1}+\lambda_{2}+\lambda_{3}$, and death rates $\min _{1 \leq i \leq n} \mu_{i} d_{i}$, representing an upper bound for the total number of customers in the studied system. Next, we explain how to apply these techniques in order to define bounding systems on subnetworks.

\section{BOUNDING SYSTEMS}

The application of bounding techniques is difficult as we have many kinds of transitions for the process due to positive and negative customers. In order to define the bounding systems, first we define the many-to-one mapping function, and we study the behavior of the subnetwork in the whole system.

\subsection{Subnetwork analysis}

Let $E=\{1, \ldots n\}$ be the index set for the nodes of the queueing system. We propose to partition the index set into disjoint index sets. In this paper, we take a partition of two index sets : $I$ which contains the nodes of the subnetwork used for the performance study, and $J$ is the complementary $J=E-I$. In order to simplify the notation, we suppose that the queues of the network are numbered beginning with the queues of the subnetwork $I$. Thus if the number of queues in the subnetwork $I$ is $k \leq n$, then $I=\{1, \ldots k\}$, and $J=\{k+1, \ldots, n\}$ is the rest of the network. We propose to define a many-to-one mapping function $g$, such that $\forall x \in \mathbb{N}^{n}, g(x)=z$, where $z$ represents the state of the subnetwork $I$, so $z \in \mathbb{N}^{k}$. Similarly to $e_{i} \in \mathbb{N}^{n}$, we denote by $v_{i} \in \mathbb{N}^{k}$ the vector with components null except the component $i$ which equals 1 . We apply Theorem 3 in order to define the bounding systems. First, we study the mapping $g$ of the process $\{X(t), t \geq 0\}$, denoted $\{g(X(t)), t \geq 0\}$, in order to apply stochastic comparison by mapping functions. Due to an event (arrival in a queue of a positive or a negative customer, a service, a transfer from a queue to another queue), the original model $\{X(t), t \geq 0\}$ evolves from state $x \in \mathbb{N}^{n}$ to a state $x^{\prime} \in \mathbb{N}^{n}$. We distinguish two cases depending on whether the events modify the subnetwork $I$ or not. If the event does not affect queues in $I$, then mapping $g(x)=g\left(x^{\prime}\right)$. Otherwise $g\left(x^{\prime}\right)$ must take into account the modification of queues of $I$ due to these events. As an example, let us consider the following two cases from state $x$ :

1. an arrival of a positive customer in queue $i$, then $x^{\prime}=$ $x+e_{i}$ and we have the following cases for the mapping $g\left(x^{\prime}\right)$ :

(a) if queue $i \notin I$ then as queue $i$ is not in the subnetwork, $g\left(x^{\prime}\right)=g(x)$.

(b) if queue $i \in I$ then $g\left(x^{\prime}\right)=g(x)+v_{i}$ (where $1 \leq$ $i \leq k)$ as queue $i$ is in the subnetwork. So this event has an impact on $g(X(t))$ because $g(x+$ $\left.e_{i}\right) \neq g(x)$.

2. a transition of a positive customer from queue $i$ to queue $j$ if $x_{i}>0$, then $x^{\prime}=x-e_{i}+e_{j}$, and we have the following cases for the mapping $g\left(x^{\prime}\right)$ :
(a) if $i \notin I$ and $j \notin I: g\left(x^{\prime}\right)=g(x)$.
(b) if $i \notin I$ and $j \in I: g\left(x^{\prime}\right)=g(x)+v_{j}$.
(c) if $i \in I$ and $j \notin I: g\left(x^{\prime}\right)=g(x)-v_{i}$.
(d) if $i \in I$ and $j \in I: g\left(x^{\prime}\right)=g(x)-v_{i}+v_{j}$.

3. a service to the outside from queue $i$ if $x_{i}>0: x^{\prime}=$ $x-e_{i}$, and we have the following cases for $g\left(x^{\prime}\right)$ :
(a) if $i \notin I g\left(x^{\prime}\right)=g(x)$.
(b) if $i \in I: g\left(x^{\prime}\right)=g(x)-v_{i}$.

4. an arrival of a negative customer to queue $i$ if $x_{i}>0$ : $x^{\prime}=x-x_{i} e_{i}$, and we have for $g\left(x^{\prime}\right)$ :
(a) if $i \notin I: g\left(x^{\prime}\right)=g(x)$.
(b) if $i \in I: g\left(x^{\prime}\right)=g(x)-x_{i} v_{i}$.

5. a transition of a negative customer from a queue $i$ to a queue $j$ if $x_{i}>0: x^{\prime}=x-e_{i}-x_{j} e_{j}$ if $x_{j}>0$, otherwise $x^{\prime}=x-e_{i}$, and we have for $g\left(x^{\prime}\right)$ :
(a) if $i \notin I$ and $j \notin I: g\left(x^{\prime}\right)=g(x)$
(b) if $i \notin I$ and $j \in I: g\left(x^{\prime}\right)=g(x)-x_{j} v_{j}$ if $x_{j}>0$.
(c) if $i \in I$ and $j \notin I: g\left(x^{\prime}\right)=g(x)-v_{i}$.
(d) if $i \in I$ and $j \in I: g\left(x^{\prime}\right)=g(x)-v_{i}-x_{j} v_{j}$ if $x_{j}>0$, otherwise : $g\left(x^{\prime}\right)=g(x)-v_{i}$.

We can now deduce how the process $g(X(t))$ evolve. In cases (1)-(a), (2)-(a), (3)-(a), (4)-(a), (5)-(a), as the events have no influence on the subnetwork then $g\left(x^{\prime}\right)=g(x)$. The events having an impact for queues in $I$ are the following:

- an arrival in queue $i \in I$ case (1)-(b), or a transition from queue $j \notin I$ to queue $i \in I$ case (2)-(b) then $g\left(x^{\prime}\right)=g(x)+v_{i}$. The transition rate is then summed for cases (1)-(b) and (2)-(b):

$$
\sum_{x^{\prime} \mid g\left(x^{\prime}\right)=g(x)+v_{i}} Q\left(x, x^{\prime}\right)=\lambda_{i}^{+}+\sum_{j \notin I} \mu_{j} P^{+}(j, i) 1_{x_{j}>0}
$$

- a transfer of a positive customer from queue $i \in I$ to queue $j \in I$ (case (2)-(d)), then $g\left(x^{\prime}\right)=g(x)-v_{i}+v_{j}$. The transition rate of this case is then:

$$
\sum_{x^{\prime} \mid g\left(x^{\prime}\right)=g(x)-v_{i}+v_{j}} Q\left(x, x^{\prime}\right)=\mu_{i} P^{+}(i, j)
$$

- a departure of a postive customer from queue $i \in I$ to the outside (case (3)-(b)) or a transfer of a positive or a negative customer from queue $i \in I$ to queue $j \notin I$ (cases (2)-(c) and (5)-(c)), then $g\left(x^{\prime}\right)=g(x)-v_{i}$. The transition rate is then summed for these cases:

$$
\begin{aligned}
\sum_{x^{\prime} \mid g\left(x^{\prime}\right)=g(x)-v_{i}} Q\left(x, x^{\prime}\right) & =\mu_{i} d_{i}+\sum_{j \notin I} \mu_{i} P^{+}(i, j) \\
& +\sum_{j \notin I} \mu_{i} P^{-}(i, j)
\end{aligned}
$$


- an arrival of a negative customer from the outside to queue $i \in I$ (case (4)-(b)) or a transfer from queue $j \notin I$ to queue $i$ (case (5)-(b)) then we have $g\left(x^{\prime}\right)=$ $g(x)-x_{i} v_{i}$. The transition rate is then summed:

$$
\sum_{x^{\prime} \mid g\left(x^{\prime}\right)=g(x)-x_{i} v_{i}} Q\left(x, x^{\prime}\right)=\lambda_{i}^{-}+\sum_{j \notin I} \mu_{j} P^{-}(j, i) 1_{x_{j}>0}
$$

- a transition of a negative customer from queue $j \in I$ to queue $i \in I$ (case (5)-(d)), then $g\left(x^{\prime}\right)=g(x)-v_{j}-x_{i} v_{i}$, where $x_{i} \geq 0$. The transition rate is :

$$
\sum_{x^{\prime} \mid g\left(x^{\prime}\right)=g(x)-v_{j}-x_{i} v_{i}} Q\left(x, x^{\prime}\right)=\mu_{j} P^{-}(j, i)
$$

We propose now to define bounding systems using the mapping function $g$ on the process $X(t)$. These bounding processes are defined on a smaller state space, so their analysis are easier than that of the original model. We propose to use the component-wise partial ordering denoted by $\preceq$ on this state space:

$$
\forall x, y \in \mathbb{N}^{n}, x \preceq y \Leftrightarrow x_{i} \leq y_{i}, \forall i=1, \ldots, n
$$

This order is widely used for multidimensional state spaces as it allows us to compare queue by queue the behavior of queueing networks. We apply the coupling by mapping functions and Theorem 3 in order to define the process $\left\{Y^{u}(t), t \geq\right.$ $0\}$ representing an upper bound for the subnetwork $I$ :

$$
\{g(X(t)), t \geq 0\} \preceq_{s t}\left\{Y^{u}(t), t \geq 0\right\}
$$

and the process $\left\{Y^{l}(t), t \geq 0\right\}$ representing a lower bound:

$$
\left\{Y^{l}(t), t \geq 0\right\} \preceq_{s t}\{g(X(t)), t \geq 0\}
$$

It is clear that G-Networks (and also G-Networks with catastrophes) are not $\preceq_{s t}$-monotone due to transitions of negative customers between queues. As an example, we suppose a network of two queues, and we try to apply the coupling of the process with itself (see theorem 4) from two comparable states $x=(0,1)$ and $y=(1,1)$ where for each of them the first component (resp. the second component) represents the number of customers in queue 1 (resp. in queue 2 ). If we suppose the order component by component, then $(0,1) \preceq(1,1)$. If we consider as an example the event of a flushing from queue 1 to queue 2 . Then from state $(0,1)$ we stay in the state $(0,1)$, while from $(1,1)$ we transit to $(0,0)$. So the order will be reversed, and the process is not monotone. As the bounding systems will be defined as GNetworks with a smaller size, then the process could be also not monotone. Therefore we could not apply the stochastic comparison by comparing the transition from the same state $x$. We propose to use the general coupling method.

\subsection{Upper bound}

We use the general coupling method by studying the coupled process $Z(t)=\left(\widehat{X}(t), \widehat{Y}^{u}(t)\right)$, such that the realizations stay in:

$$
K=\left\{(x, y) \in N^{n} \times N^{k} \mid g(x) \preceq y\right\} .
$$

where $\widehat{X}(t)$ and $\widehat{Y}^{u}(t)$ are also Markov processes with infinitesimal generators $Q$ and $Q^{u}$. The process $Z(t)$ is called a coupling process associated to $K$. Outside of $K$, the two coordinates may move independently, but in $K$ the jumps of the two coordinates cannot be independent if we want to remain in $K$. As an example, if we go out of $K$ by a jump with the first component, then we must compensate it with the jump of the second component to remain in $K$. For states $(x, y) \in K$, if $x$ transits to $x^{\prime}$ such that $\left(x^{\prime}, y\right) \notin K$ (if $\left.g\left(x^{\prime}\right) \npreceq y\right)$, then the second component $y$ must compensate by a transit to $y^{\prime}$ such that $\left(x^{\prime}, y^{\prime}\right) \in K\left(g\left(x^{\prime}\right) \preceq y^{\prime}\right)$. Similarly, we have to consider how we go out of $K$ by a jump with the second component. Note that states $(x, y) \in K$ where the transition from $x$ to $x^{\prime}$ is such that $\left(x^{\prime}, y\right) \notin K$, or a transition from $y$ to $y^{\prime}$ such that $\left(x, y^{\prime}\right) \notin K$ can occur are called the frontier states. So the frontier states are the states $(x, y) \in K$ such that $x_{i}=y_{i}, i \in\{1, \cdots, k\}$. By taking into account the events happening in $\{X(t), t \geq 0\}$, triggering a transition from $x$ to $x^{\prime}$ we compute the transition rates of $\left\{Y^{u}(t), t \geq 0\right\}$ such that the transitions of the coupled process $\{Z(t), t \geq 0\}$ remain in $K$. The transition rates of $Q^{u}$ are then computed using the coupling by the mapping function $g$ and the set $K$.

Next, we present depending on events how a jump of a component must be compensated by the jump of the other component, in order to obtain the conditions on the transition rates. Note that the jump of component $x$ makes the coupled process can go out of $K$, if an arrival occurs, and so must be compensated by an equivalent jump of $y$. The jumps of $y$ that makes the coupled process leave $K$ are services or flushing of queues, and must be compensated by similar jumps of $x$. We now consider the different events happening in a queue $i$ in order to analyse the jumps of the components:

1. If we have an arrival in a queue $i \in I$ troggering a transition from state $x$ to $x^{\prime}$ such that $g\left(x^{\prime}\right)=g(x)+v_{i}$, then we must have also a transition from $y$ to $y^{\prime}$ such that $y^{\prime}=y+v_{i}$, in order to obtain $g\left(x^{\prime}\right) \preceq y^{\prime}$. Thus transition rates must satisfy the following condition:

$$
Q^{u}\left(y, y+v_{i}\right) \geq \sum_{x^{\prime} \mid g\left(x^{\prime}\right)=g(x)+v_{i}} Q\left(x, x^{\prime}\right)
$$

Since inequality (9) must be satisfied for all states $x$ such that $(x, y) \in K$, then we take the maximum of the right part over $x \in \mathbb{N}^{n}$ :

$$
Q^{u}\left(y, y+v_{i}\right)=\max _{x \mid g(x) \preceq y}\left\{\sum_{x^{\prime} \mid g\left(x^{\prime}\right)=g(x)+v_{i}} Q\left(x, x^{\prime}\right)\right\}
$$

The evolution of $\{g(X(t)), t \geq 0\}$ due to this event $\left(Q\left(x, x^{\prime}\right)\right)$ is given in equation $(2)$, and the maximum of this rate is reached for states $x$ such that $x_{j}>$ $0, \forall j \notin I$. Thus we choose $Q^{u}\left(y, y+v_{i}\right)=\lambda_{i}^{+}+$ $\sum_{j \notin I} \mu_{j} P^{+}(j, i)$.

2. The transition from a queue $i$ to a queue $j$ can trigger a jump out of $K$ for the first or the second component. For the first component, if we have a transition between $x$ to $x^{\prime}$ such that $g\left(x^{\prime}\right)=g(x)-v_{i}+v_{j}$, then we must have also a transition from $y$ to $y^{\prime}$ such that $y^{\prime}=y-v_{i}+v_{j}$ or a transition such that $y^{\prime}=y+v_{j}$. For the second component, if we have a transition from $y$ to $y^{\prime}$ such that $y^{\prime}=y-v_{i}+v_{j}$, then we must have a transition from $x$ to $x^{\prime}$ such that $g\left(x^{\prime}\right)=$ $g(x)-v_{i}+v_{j}$ or $g\left(x^{\prime}\right)=g(x)-v_{i}$. As the jumps 
for services and arrivals are used to compensate these events, we take only the transit between queues $i$ and $j$. It results the same transition rate, so from equation (3): $Q^{u}\left(y, y-v_{i}+v_{j}\right)=\mu_{i} P^{+}(i, j)$.

3. If we have a transit of a negative customer from queue $i$ to queue $j$ making a transition from $y$ to $y^{\prime}$ such that $y^{\prime}=y-v_{i}-y_{j} v_{j}$, then we must have also a transition from $x$ to $x^{\prime}$ such that $g\left(x^{\prime}\right)=g(x)-v_{i}-x_{j} v_{j}$ if $x_{i}>0$. As this event occurs in the exact system only if $x_{i}>0$, then we take the minimum rate: $Q^{u}\left(y, y-v_{i}-y_{j} v_{j}\right)=$ 0 , in order to avoid to have 0 in the component $y_{j}$ (if $y_{i}>0$ ) while $x_{j}$ could not be modified (if $x_{i}=0$ ).

4. If we have a service in a queue $i \in I$ making a transition from $y$ to $y^{\prime}$ such that $y^{\prime}=y-v_{i}$, then we must have a transition from $x$ to $x^{\prime}$ such that $g\left(x^{\prime}\right)=g(x)-v_{i}$, so we have the following inequality:

$$
Q^{u}\left(y, y-v_{i}\right) \leq \sum_{x^{\prime} \mid g\left(x^{\prime}\right)=g(x)-v_{i}} Q\left(x, x^{\prime}\right)
$$

As this inequality must be verified for all $(x, y) \in K$, then we take:

$$
Q^{u}\left(y, y-v_{i}\right)=\min _{x \mid g(x) \preceq y}\left\{\sum_{x^{\prime} \mid g\left(x^{\prime}\right)=g(x)-v_{i}} Q\left(x, x^{\prime}\right)\right\}
$$

From equation (4), we take: $Q^{u}\left(y, y-v_{i}\right)=\mu_{i} d_{i}+$ $\sum_{j \notin I} \mu_{i} P^{+}(i, j)+\sum_{j \notin I} \mu_{i} P^{-}(i, j)$. Moreover, as in the precedent case we have supposed that $Q^{u}(y, y-$ $\left.v_{i}-y_{j} v_{j}\right)=0(i, j \in I)$ we consider in the upper bound that the rate $\mu_{i} P_{i j}^{-}$has no impact in component $j$, and serves only to decrease the component $i$. So we obtain $Q^{u}\left(y, y-v_{i}\right)=\mu_{i} d_{i}+\sum_{j \notin I} \mu_{i} P^{+}(i, j)+$ $\sum_{j \notin I} \mu_{i} P^{-}(i, j)+\sum_{j \in I} \mu_{i} P^{-}(i, j)$.

5. If we have an external arrival of a negative customer to a queue $i \in I$ triggering a transition from $y$ to $y^{\prime}$ such that $y^{\prime}=y-y_{i} v_{i}$, then we must have a transition from $x$ to $x^{\prime}$ such that $g\left(x^{\prime}\right)=g(x)-x_{i} v_{i}$. For compensating, we could have also a transition from $x$ to $x^{\prime}$ such that $g\left(x^{\prime}\right)=g(x)-v_{j}-x_{i} v_{i}$ (for $j \neq i$ and $j \in I$ ), in the case of $x_{j}>0$. For the two cases, we take the minimum rates obtained when $x_{j}=0, \forall j \neq i$, so from equations (5) we obtain $Q^{u}\left(y, y-v_{i}\right)=\lambda_{i}^{-}$

Thus for a queue $i \in I$, the transition rates are :

- arrival rate $\lambda_{i}^{+}+\sum_{j \notin I} \mu_{j} P^{+}(j, i)$.

- transition rate to queue $j \in I: \mu_{i} P^{+}(i, j)$.

- departure rate : $\mu_{i} d_{i}+\sum_{j \notin I} \mu_{i} P^{+}(i, j)+\sum_{j \neq i} \mu_{i} P^{-}(i, j)$.

- arrival rate of a signal (negative customer) from the outside : $\lambda_{i}^{-}$.

As the rates of $\left\{Y^{u}(t), t \geq 0\right\}$ are defined such that the coupled process stay in the set $K$, then equation (7) is satisfied. For the upper bound, the transition rates $\mu_{j} P^{+}(j, i)$ of positive customers coming from the rest of the network are added to the external arrival rates $\lambda_{i}^{+}$of positive customers.
The departure rate is the rate of the departure from the subnetwork. For negative customers, only external arrival rates are considered. Inside the subnetwork the transit of positive customers is kept, but not the transition of negative customers which is considered as a departure rate. Intuitively speaking, any queue of the upper bound has more positive customers, but is less flushed than in the exact system. We can also remark that the upper bound is the most accurate bound that we could obtain from the constraints inequalities. Moreover as the transitions happen for any state, then the bounding model is monotone (see theorem 4).

\subsection{Lower bound}

Similarly to the upper bound case, we apply Theorem 3 in order to define $\left\{Y^{l}(t), t \geq 0\right\}$ providing a lower bound:

$$
\forall t \geq 0, \quad Y^{l}(t) \preceq_{s t} g(X(t))
$$

We will define the infinitesimal generator $Q^{l}$ such that the Theorem 3 is verified, which means that we study the coupled process $Z(t)=\left(\left(Y^{l}(t), X(t)\right)\right.$, such that realisations stay in:

$$
K=\left\{(x, y) \in \mathbb{N}^{k} \times \mathbb{N}^{n} \mid x \preceq g(y)\right\}
$$

We study all events from state $y$ in the process $X(t)$ such that $g(y)$ varies.

1. If we have an arrival in a queue $i \in I$ generating a transition from $x$ to $x^{\prime}$ such that $x^{\prime}=x+v_{i}$, then we must have also a transition from $y$ to $y^{\prime}$ such that $g\left(y^{\prime}\right)=g(y)+v_{i}$. Then we must have:

$$
Q^{l}\left(x, x+v_{i}\right) \leq \sum_{y^{\prime} \mid g\left(y^{\prime}\right)=g(y)+v_{i}} Q\left(y, y^{\prime}\right)
$$

By considering the minimum rate in equation $(2)\left(x_{j}=\right.$ $0, \forall j \notin I)$, we take $Q^{l}\left(x, x+v_{i}\right)=\lambda_{i}^{+}$.

2. As in the case (2) of the upper bound, we take the same transition rate: $Q^{l}\left(x, x-v_{i}+v_{j}\right)=\mu_{i} P^{+}(i, j)$.

3. If we have a service in a queue $i \in I$ from $y$ then we must have also a decrease of the number of customers of queue $i$ in $x$ : (a service in the same queue $i$ from $x$ or a transit of a positive or a negative customer from queue $i$ to any queue $j \notin I$. So from equation (4), we take:

$$
Q^{l}\left(x, x-v_{i}\right)=\mu_{i} d_{i}+\sum_{j \notin I} \mu_{i} P^{+}(i, j)+\sum_{j \notin I} \mu_{i} P^{-}(i, j) .
$$

4. If we have a transition from queue $i$ to queue $j$ triggering a transition from $y$ to $y^{\prime}$ such that $g\left(y^{\prime}\right)=$ $g(y)-v_{i}-y_{j} v_{j}$ then we can have a transition from $x$ to $x^{\prime}$ such that $x^{\prime}=x-v_{i}-x_{j} v_{j}$ if $x_{i}>0$ or $x^{\prime}=x-x_{j} v_{j}$ if $x_{i}=0$.

if $x_{i}>0$ :

$$
\sum_{y^{\prime} \mid g\left(y^{\prime}\right)=g(y)-v_{i}-y_{j} v_{j}} Q\left(y, y^{\prime}\right) \leq Q^{l}\left(x, x-v_{i}-x_{j} v_{j}\right)
$$

If we replace the first part of the inequality by the rate given in equation (6), then we obtain:

$$
Q^{l}\left(x, x-v_{i}-x_{j} v_{j}\right)=\mu_{i} P^{-}(i, j)
$$




$$
\begin{aligned}
& \text { If } x_{i}=0: \\
& \sum_{y^{\prime} \mid g\left(y^{\prime}\right)=g(y)-v_{i}-y_{j} v_{j}} Q\left(y, y^{\prime}\right) \leq Q^{l}\left(x, x-x_{j} v_{j}\right)
\end{aligned}
$$

This relation will be important to define the external flushing rate to queue $j$. We have the condition for all $i \neq j \in I$ such that $x_{i}=0$ :

$$
Q^{l}\left(x, x-x_{j} v_{j}\right) \geq \mu_{i} P^{-}(i, j) .
$$

And we derive :

$$
Q^{l}\left(x, x-x_{j} v_{j}\right) \geq \sum_{i \in I, i \neq j} \mu_{i} P^{-}(i, j) 1_{x_{i}=0}
$$

5. If we have an arrival of a negative customer in queue $i \in I$ making a transition from $y$ to $y^{\prime}$ such that $g\left(y^{\prime}\right)=$ $g(y)-y_{i} v_{i}$, then we could have a transition from $x$ to $x^{\prime}$ such that $x^{\prime}=x-x_{i} v_{i}$ or $x^{\prime}=x-x_{j}-x_{i} v_{i} \forall j \in I$ if $x_{j}>0$. As the second transition is already used to compensate the transition from $y$ to $y^{\prime}$ such that $g\left(y^{\prime}\right)=g(y)-v_{i}-y_{j} v_{j}$ (in the precedent case), then we take only the transition to $x-x_{i} v_{i}$. From equations (5), we take the maximum value by considering $x_{j}>0$, $\forall j \neq I$. Moreover, by considering also inequality (10) where we consider the flushing of queue $i$ instead of queue $\mathrm{j}$, we add the sum of transition rate $\mu_{j} P^{-}(j, i)$ for the case where queues $j$ are idle to the external flushing rate of queue $i$, and we obtain the following value :

$$
\begin{gathered}
Q^{l}\left(x, x-x_{i} v_{i}\right)=\lambda_{i}^{-}+\sum_{j \notin I} \mu_{j} P^{-}(j, i) \\
+\sum_{j \in I, j \neq i} \mu_{j} P^{-}(j, i) 1_{x_{j}=0}
\end{gathered}
$$

Thus for a queue $i \in I$, the transition rates are :

- arrival rate: $\lambda_{i}^{+}$.

- transit rate to queue $j \in I: \mu_{i} P^{+}(i, j)$.

- departure rate: $\mu_{i} d_{i}+\sum_{j \notin I} \mu_{i} P^{+}(i, j)+\sum_{j \notin I} \mu_{i} P^{-}(i, j)$.

- transit rate of a signal (negative customer) to queue $j$ : $\mu_{i} P^{-}(i, j)$.

- arrival rate of a signal:

$$
\lambda_{i}^{-}+\sum_{j \notin I} \mu_{j} P^{-}(j, i)+\sum_{j \in I, j \neq i} \mu_{j} P^{-}(j, i) 1_{x_{j}=0} .
$$

As the rates of $\left\{Y^{l}(t), t \geq 0\right\}$ are defined such that the coupled process stays in the set $K$, then equation (8) is satisfied. For the lower bound, we remark that transition rates $\mu_{j} P^{-}(j, i)$ of flows of negative customers coming from the rest of the network are added to the external flows of negative customers. The departure rate is the rate of the departure to the outside of the subnetwork. For arrivals of positive customers, only external arrival rates of positive customers are considered. Inside the subnetwork the transit of positive and negative customers are kept. Moreover, the rate $\mu_{i} P^{-}(i, j)$ of negative customers between queues is considered in the lower bound for any value of $x_{i}$ : if $x_{i}>0$, and if $x_{i}=0$ it is added to the external signal arrival rate. So with this rate, a queue $j$ is always flushed whatever the state of the other queues. We can remark also that this system is the more accurate lower bound for the subnetwork, and as the transitions trigger whatever the state is, the lower bound is monotone (see theorem 4). Intuitively, for the lower bound each queue has less positive customers, and is more flushed than in the exact process.

\subsection{Transient measure comparisons}

In this section, we identify which measures could be compared between G-Network with catastrophes and bounding systems. Obviously, we have the comparison of the mean number of customer in queue $i \in I$ at any time $t$. GNetworks are very efficient models for software virus infections [14]. In G-network with catastrophes, flushes of queues can represent malware infections which cause the deletion of informations (represented by positive customers) in the stations. Several interesting performance measures can be computed using bounding techniques. Let $N_{i}(t)$, be the number of times a station $i$ of the subnetwork $I$ has been infected in the time interval $[0, t]$. We denote by $N_{i}^{u}(t)$ (resp. $\left.N_{i}^{l}(t)\right)$ the corresponding measure for $\left\{Y^{u}(t), t \geq 0\right\}$ (resp. $\left.\left\{Y^{l}(t), t \geq 0\right\}\right)$. Then we have the following comparison :

$$
\begin{aligned}
& \text { Proposition 1. } \forall t \geq 0, \forall i \in I: \\
& N_{i}(t) \leq_{s t} N_{i}^{l}(t), \text { and } N_{i}^{u}(t) \leq_{s t} N_{i}(t)
\end{aligned}
$$

Proof. We use the coupling of the processes with the flush event, as it is the only event which modifies the behaviour of these processes. We define the process $\left\{\widehat{N}_{i}(t), t \geq\right.$ $0\}$ (resp. $\left\{\widehat{N}_{i}^{l}(t), t \geq 0\right\}$ ) with the same transition rates as $\left\{N_{i}(t), t \geq 0\right\}$ (resp. $\left.\left\{N_{i}^{l}(t), t \geq 0\right\}\right)$, with the following initial condition: $\widehat{N}_{i}(0)[\omega] \leq \widehat{N}_{i}^{l}(0)[\omega]$. For the proof, we suppose that:

$$
\widehat{N}_{i}(t)[\omega] \leq \widehat{N}_{i}^{l}(t)[\omega]
$$

and we will prove that this inequality is also satisfied at time $t+d t$. As we have proved that the processes $\{g(X(t)), t \geq 0\}$ and $\left\{Y^{l}(t), t \geq 0\right\}$ are $\preceq_{s t}$-comparable, then it means that the flush event occurs more in $\left\{Y^{l}(t), t \geq 0\right\}$ then in the corresponding subnetwork of the exact system, so if $\widehat{N}_{i}(t)$ increases then $\widehat{N}_{i}^{l}(t)$ increases also and equation (11) is verified at time $t+d t$, so $N_{i}(t) \leq_{s t} N_{i}^{l}(t)$. Similarly, we can prove that $N_{i}^{u}(t) \leq_{s t} N_{i}(t)$.

Another interesting measure that can be computed, is the time until the first infection of a queue $i$ in a subnetwork $I$. If $T f_{i}$ denotes the time until the first flush, then we deduce the following inequality :

\section{Proposition 2.}

$$
\forall i \in I, T f_{i} \leq_{s t} T f_{i}^{u} \text { and } T f_{i}^{l} \leq_{s t} T f
$$

ProOF. Let $\widehat{T f}_{i}\left(\operatorname{resp} \widehat{T f}_{i}^{u}\right)$ be the random variable with the same probability distribution then $T f_{i}\left(\right.$ resp. $\left.T f_{i}^{u}\right)$. We 
suppose the case where no flush has occurred yet in the interval $[0, t]$ for both systems. It is clear that when a flush occurs in a queue $i$ of $Y^{u}(t)$, then it has already occurred in $X(t)$. So we deduce that : $\widehat{T f}_{i}[\omega] \preceq \widehat{T f}_{i}^{u}[\omega], \forall \omega \in \Omega$. Similarly, we can prove that $T f_{i}^{l} \leq_{s t} T f_{i}$, and proposition 2 is proved.

\section{NUMERICAL RESULTS}

We present some transient performance measures results in order to illustrate the proposed approach. We study a Gnetwork with 7 queues represented in Figure 1. We focus on queue 7 in order to compute the transient performance measures from different subnetworks. Three subnetworks are studied : $I_{1}=\{7\}, I_{2}=\{5,7\}$, and $I_{3}=\{5,6,7\}$ (where $\left.I_{1} \subset I_{2} \subset I_{3}\right)$ in order to study the accuracy of the bounds. The results are obtained using the Matlab/Simevent simulator. About 100 sample paths have been generated in order to obtain with $95 \%$ confidence the indifference region with width of 0.01 of the estimated value. We take the following values for the parameters : for $1 \leq i \leq 6: \lambda_{i}^{+}=2$, $\lambda_{7}^{+}=3$, and for $1 \leq i \leq 7: \lambda_{i}^{-}=-\frac{1}{25}, \mu_{i}=2$. The non null probabilities are as follows: $P^{+}(1,3)=P^{+}(1,4)=0.3$, $P^{-}(1,3)=P^{-}(1,4)=0.1, d_{1}=0.2, P^{+}(2,4)=0.6$, $P^{-}(2,4)=0.2, d_{2}=0.2, P^{-}(3,5)=P^{-}(3,6)=0.1$, $P^{+}(3,5)=P^{+}(3,6)=0.3, d_{3}=0.2, P^{+}(4,6)=P^{-}(4,6)=$ $0.4, d_{4}=0.2, P^{+}(5,7)=0.6, P^{-}(5,7)=0.2, d_{5}=0.2$, $P^{+}(6,7)=0.3, P^{-}(6,7)=0.5, d_{6}=0.2$.

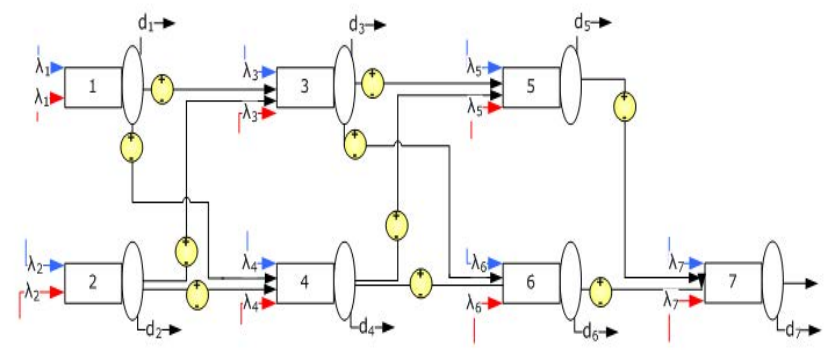

Figure 1: The G-Network understudy.

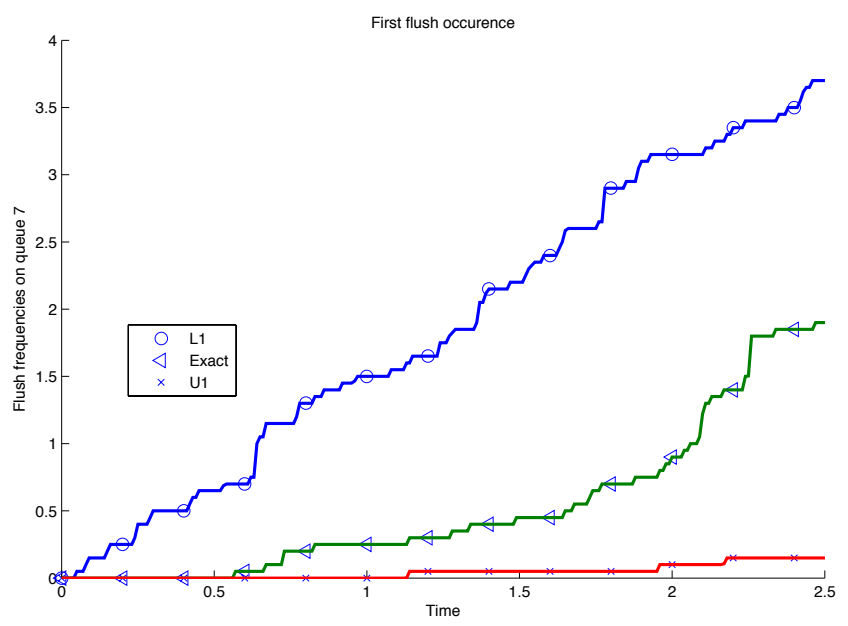

Figure 2: Time until first failure in queue 7 .

\begin{tabular}{|l|l|}
\hline System & Simulation time \\
\hline Exact & $363 \mathrm{~s}$ \\
\hline$U_{3}$ & $149 \mathrm{~s}$ \\
\hline$U_{2}$ & $100 \mathrm{~s}$ \\
\hline$U_{1}$ & $53 \mathrm{~s}$ \\
\hline
\end{tabular}

Table 1: Simulation times in seconds

For all the figures, we denote by $L_{1}, L_{2}, L_{3}$ (resp. $U_{1}, U_{2}$, $U_{3}$ ) the lower bounds (resp. the upper bounds) obtained from the subnetworks $I_{1}, I_{2}, I_{3}$. In Figure 2, we study the time until the first failure $T f_{7}$, and we give the lower bound $L_{1}$, the exact value obtained from the queueing network with 7 queues, and the upper bound $U_{1}$. We deduce from Figure 2 that the first failure occurs in queue 7 before a time equal approximately to 1.12 .

In Figure 3, we represent the number of customers in queue 7 at any time $t \in[0,10]$. Obviously, $U_{1}$ is the worst upper bound as it is represented by only queue 7 , and $U_{3}$ provides the best upper bound as it is represented by a larger network. On the other hand, the lower bounds provide the same results which is obvious as queue 7 has similar rates in $L_{1}, L_{2}$, and $L_{3}$. We can easily remark that the lower bound is close to the exact value, while the upper bounds are far from it especially when the time increases. This is because in the upper bounds, we have not considered the transition of negative customers between queues, so the behaviour is relatively far from the exact system, especially when the time increases. On the other side, the lower bound has a good accuracy as we have considered both positive and negative customers in the system.

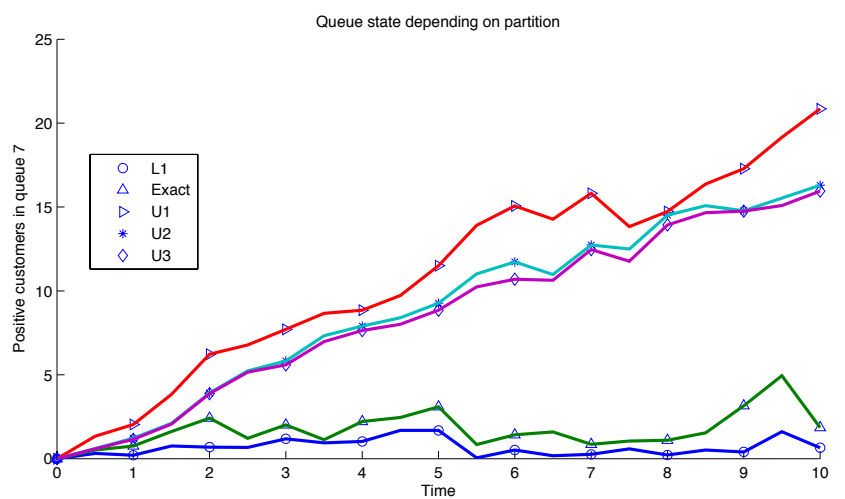

Figure 3: Number of positive customers in queue 7 for $\mu_{5}=\mu_{6}=2$, and $t \in[0,10]$.

In Figure 4 , we take $\mu_{5}=\mu_{6}=6$, in order to reduce the load of queues connected to queue 7 , and we consider a smaller time interval. We can see in this case, that upper bounds are closer to the exact value, and also the gap between the bounds is significant. Moreover, if we consider also the simulation times given in Table 1, we can see the relevance of our approach. Instead of computing the exact values with a simulation time of 363 seconds, we obtain bounding values with a tradeoff between accuracy of bounds and simulation time. 


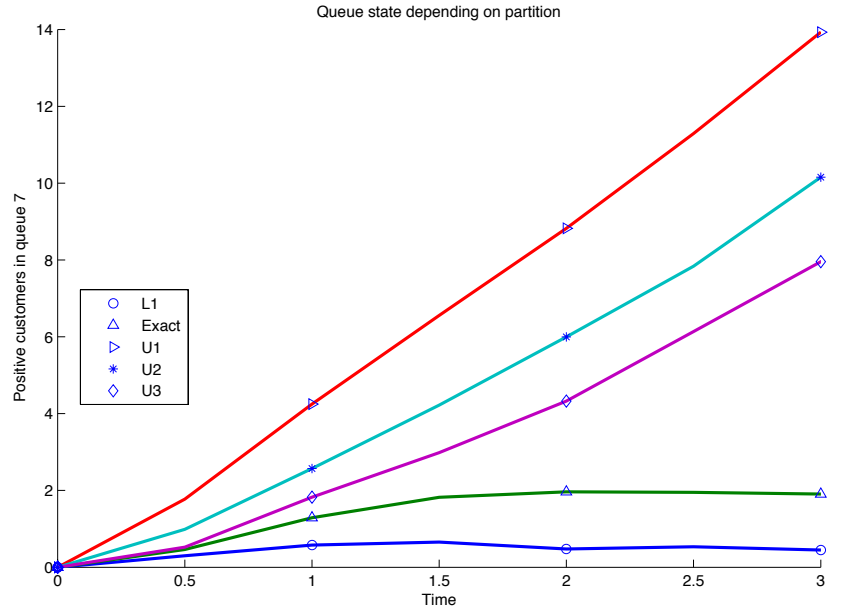

Figure 4: Number of positive customers in queue 7 for $\mu_{5}=\mu_{6}=6$, and $t \in[0,3]$.

\section{CONCLUSION}

We have seen in this paper that application of stochastic comparisons for transient analysis of G-networks with catastrophes provides an interesting solution as we obtain a tradeoff between the quality of the bounds and the computational complexity. As a future work, it is interesting to define a general algorithm based on the coupling by mapping functions in order to analyse transient behaviour of other networks. We will also study how we can extend this stochastic comparison approach to more abstracted representations such as the tensor based representation of Master-Slave synchronisation with product form introduced in [8] or Stochastic Process Algebra.

\section{REFERENCES}

[1] J.R. Artalejo. G-networks: a versatile approach for work removal in queueing networks. European Journal Operational Research, 126:233-249, 2000.

[2] G. Bolch, S. Greiner, H.de Meer, and K.S. Trivedi. Queueing Networks and Markov Chains. Wiley-Interscience, 2006.

[3] H. Castel, L. Mokdad, and N. Pekergin. Aggregated bounding Markov processes applied to the analysis of tandem queues. In ValueTools 2007, page 43, Nantes, France, 23-25, October 2007. ACM.

[4] H. Castel-Taleb, J-M. Fourneau, and N. Pekergin. Stochastic comparisons applied to G-networks with catastrophes. In ISCIS 2010, London, UK, 22-24, September 2010. LNEE, Springer.

[5] H. Castel-Taleb, I. Ismael-Aouled, and N. Pekergin. Bounding aggregations for transient and stationary performance analysis of subnetworks. Computer Journal (to appear), 2011.

[6] X. Chao. A queueing network model with catastrophes and product form solution. Operations Research Letters, 18:75-79, 1995.

[7] A. Crescenzo, V. Giorno, A.G. Nobile, and LM. Ricciardi. A note on birth-death processes with. Statistics and Probability Letters, 78:2248-2257, 2008.
[8] T. Dao-Thi and J-M. Fourneau. Stochastic automata networks with master/slave synchronization: Product form and tensor. In Analytical and Stochastic Modeling Techniques and Applications, 16th International Conference, ASMTA, Madrid, Spain, 2009. LNCS.

[9] M. Doisy. Coupling technique for comparison of functions of Markov processes. Journal of Applied Mathematics and Decision Sciences, 4:131-154, 2000.

[10] E. Gelenbe. Product form queueing networks with negative and positive customers. Journal of Applied Probability, 28:656-663, 1991.

[11] E. Gelenbe. G-networks with instantaneous customer movement. Journal of Applied Probability, 30(3):742-748, 1993.

[12] E. Gelenbe. G-networks with signals and batch removal. Probability in the Engineering and Informational Sciences, 7:335-342, 1993.

[13] E. Gelenbe. G-networks: An unifying model for queueing networks and neural networks. Annals of Operations Research, 48(1-4):433-461, 1994.

[14] E. Gelenbe. Dealing with software viruses : a biological paradigm. Technical Report 12, Information Security Technical report, 2007.

[15] E. Gelenbe and J-M. Fourneau. G-networks with resets. Performance Evaluation, 49(1/4):179-191, 2002.

[16] T. Kamae, U. Krengel, and G L. O'Brien. Stochastic inequalities on partially ordered spaces. The Annals of Probability, 5(6):899-912, 1977.

[17] B. Krishna Kumar and D. Arivudainambi. Transient solution of an $\mathrm{M} / \mathrm{M} / 1$ queue with catastrophes. Computers and Mathematics with Applications, 40(10-11):1233-1240, 2000.

[18] L. Lindvall. Stochastic monotonicities in Jackson queueing networks. Prob. in the Engineering and Informational Sciences, 11:1-9, 1997.

[19] T. Lindvall. Lectures on the coupling method. Wiley series in Probability and Mathematical Statistics, 1992.

[20] W. Massey. A family of bounds for the transient behaviour of a Jackson network. Journal of Applied Probability, 23:543-549, 1986.

[21] W. Massey. Stochastic orderings for Markov processes on partially ordered spaces. Mathematics of Operations Research, 12(2):350-367, 1987.

[22] A. Muller and D. Stoyan. Comparison methods for stochastic models and risks. J. Wiley \& Sons in Probability and Statistics, 2002.

[23] N. Pekergin and H. Taleb-Castel. Stochastic bounds on the transient behaviors of the G-Network. Studia Informatica, 23, 2002.

[24] M. Pinedo, X. Chao, and M. Miyazawa. Queueing Networks: Customers, Signals and Product Form Solutions. J. Wiley, 1999.

[25] M. Shaked and J.G. Shantikumar. Stochastic orders and their applications. Academic Press, Boston, 1994.

[26] L. Truffet. Reduction technique for discrete time Markov chains on totally ordered space using stochastic comparisons. Journal of Applied Probability, 37(3):795-806, 2000. 